\title{
Longitudinal vibrations of a cylindrical shell filled with a viscous compressible liquid
}

\author{
Khayrulla Khudoynazarov", and Burxon Yalgashev \\ Samarkand State University, Samarkand, Uzbekistan
}

\begin{abstract}
This article investigates the longitudinal vibrations of a semiinfinite circular cylindrical elastic shell filled with a viscous compressible fluid. It is believed that the vibrations are excited by a suddenly switched on longitudinal displacement at the end. To solve the problem, the refined equations of longitudinal vibrations of a circular cylindrical elastic shell interacting with an internal viscous compressible fluid, previously proposed by the authors, were taken as the main resolving equations. In this case, the lateral surfaces of the shell are considered free from external loads; in addition, considering purely longitudinal vibrations, it can be assumed that the radial displacements of the points of the shell are equal to zero.
\end{abstract}

\section{Introduction}

The dynamic theory of shells is one of the sections of the three-dimensional theory of elasticity, in which such problems of calculating shells are considered, under which the boundary conditions on the lateral surfaces are specified in stresses in the form of external loads. In this case, the boundary conditions on the lateral surfaces are set in stresses in the form of external loads [1]. Usually, problems about transient processes in shells and in plates and rods are usually carried out based on approximate equations of oscillation [2-5]. Therefore, the basic relations of the theory of shells are developed by reducing a threedimensional problem in spatial coordinates to a two-dimensional one, using various methods and approaches using hypotheses and premises [6]. Depending on the factors taken into account, the methods of the derivation of the approximate equations of vibration, based on the dynamic theory of elasticity, are divided into several directions [7].

One of these areas is the method of using general solutions in transformations of threedimensional problems of the dynamic theory of elasticity, which was developed in the works of IG Filippov et al. [8, 9]. In this case, in [8], approximate equations of transverse and longitudinal-radial vibrations of a cylindrical shell were constructed, and in [9], the questions of formulating the boundary conditions to them were discussed. This method has been successfully applied in the works of $\mathrm{Kh}$. Khudoinazarov et al. To the problems of unsteady vibrations of three-layer cylindrical shells [10] and plates [11, 12], in which general equations of vibrations of layered plates and shells were obtained taking into account the viscoelastic properties of the material.

\footnotetext{
*Corresponding author: kh.khudoyn@gmail.com
} 
The refined equations of vibrations of shells and plates were applied in the works of RI Khalmuradov to solve the problem of transverse vibrations of a reinforced plate [13] and frequency analysis of longitudinal-radial vibrations of a cylindrical shell [14]. Based on the solution of the obtained frequency equations, the frequencies of natural vibrations of the shell are determined. A comparative frequency analysis of longitudinal vibrations of a circular cylindrical elastic shell is carried out based on the classical Kirchhoff-Love theory, refined theories of Hermann-Mirsky, and Filippov-Khudoinazarov. Based on the results obtained, conclusions were made regarding the applicability of the studied oscillation equations, depending on the waveform and shell length.

It should be emphasized that the essence of this method for deriving the oscillation equations is reduced to studying the constructed solutions $[15,16]$ for various types of external influences and to fin-ding out the conditions under which the displacements or their "main parts" satisfy the simple oscillation equations, and to finding the algorithm, allowing for the field of these "main parts" to calculate the approximate values of the fields of displacements and stresses in any section for an arbitrary moment in time.

In recent decades, the study of non-stationary interaction of structural elements [17] and, in particular, cylindrical shells [18-20] with a viscous fluid are becoming increasingly important. In these works, various theories of vibrations of plates and shells interacting with a fluid are presented, and an analysis of their applicability in solving various problems of wave dynamics, including transient deformation processes. This article is devoted to the study of longitudinal vibrations of a cylindrical shell interacting with an internal viscous compressible fluid.

\section{Methods}

It is believed that the cylindrical shell under consideration, as a cylindrical layer (threedimensional solid), strictly obeys the mathematical linear theory of viscoelasticity and, in its exact formulation, which is described by its three-dimensional equations. Moreover, it is assigned to a cylindrical coordinate system $(r, \theta, z)$, where is the axis $z$-directed along the axis of symmetry of the cylinder. It is assumed that the vibrations of the layer, like fluids, are small. In this case, the smallness of the oscillations implies the smallness of the displacements of the points of the layer and the fluid.

\subsection{Basic equations and formulas for stress-strain state}

To solve the problem, we apply the refined oscillation equations derived in [6] for nonstationary longitudinal-radial oscillations of a circular cylindrical elastic shell, which in the absence of external influences take the form

$$
\begin{gathered}
{\left[q_{1}+\frac{\mu^{\prime}}{\mu}\left(\frac{\partial}{\partial t}-\frac{\rho_{0}^{\prime} a_{0}^{2}}{\mu^{\prime}}\right)\right] U_{r, 0}+\left[1+q_{1}+\frac{2}{3} \frac{\mu^{\prime}}{\mu}\left(\frac{\partial}{\partial t}-\frac{3}{2} \frac{\rho_{0}^{\prime} a_{0}^{2}}{\mu^{\prime}}\right)\right] \frac{\partial U_{z, 0}}{\partial z}+} \\
+\frac{r_{1}}{2}\left[\lambda_{2}+\frac{4}{r_{1}^{2}} \frac{\mu^{\prime}}{\mu}\left(\frac{\partial}{\partial t}-\frac{\rho_{0}^{\prime} a_{0}^{2}}{2 \mu^{\prime}}\right)-\frac{4}{r_{1}^{2}}\right] U_{r, 1}-\frac{r_{1}}{2}\left[1+\frac{2}{3} \frac{\mu^{\prime}}{\mu}\left(\frac{\partial}{\partial t}-\frac{3}{2} \frac{\rho_{0}^{\prime} a_{0}^{2}}{\mu^{\prime}}\right)\right] \frac{\partial U_{z, 1}}{\partial z}=0 ; \\
{\left[1+q_{1}+\frac{2}{3} \frac{\mu^{\prime}}{\mu}\left(\frac{\partial}{\partial t}-\frac{3}{2} \frac{\rho_{0}^{\prime} a_{0}^{2}}{\mu^{\prime}}\right)\right] \frac{\partial U_{r, 0}}{\partial z}+\left[\left(1-q_{1}\right) \lambda_{1}-\frac{\rho_{0}^{\prime}}{3 \mu}\left(3 a_{0}^{2} \frac{\partial^{2}}{\partial z^{2}}+4 v^{\prime} \frac{\partial^{3}}{\partial t \partial z^{2}}-3 \frac{\partial^{2}}{\partial t^{2}}\right)\right] U_{z, 0}-}
\end{gathered}
$$




$$
\begin{gathered}
-\frac{2}{r_{1}}\left[1-\frac{2 \mu^{\prime}}{3 \mu}\left(\frac{\partial}{\partial t}-\frac{3}{2} \frac{\rho_{0}^{\prime} a_{0}^{2}}{\mu^{\prime}}\right)\right] \frac{\partial U_{r, 1}}{\partial z}-\left[\frac{2}{r_{1}}+\frac{r_{1} \rho_{0}^{\prime}}{6 \mu}\left(3 a_{0}^{2} \frac{\partial^{2}}{\partial z^{2}}+4 v^{\prime} \frac{\partial^{3}}{\partial t \partial z^{2}}-3 \frac{\partial^{2}}{\partial t^{2}}\right)\right] U_{z, 1}=0 ; \\
q_{1} U_{r, 0}+\left(1+q_{1}\right) \frac{\partial U_{z, 0}}{\partial z}+r_{1}\left[\lambda_{2}+\frac{2}{r_{2}^{2}}\right] U_{r, 1}-r_{1} \frac{\partial U_{z, 1}}{\partial z}=0 ; \\
\left(1+q_{1}\right) \frac{\partial U_{r, 0}}{\partial z}+\left(1-q_{1}\right) \lambda_{1} U_{z, 0}-r_{1} \frac{2}{r_{2}^{2}} \frac{\partial U_{r, 1}}{\partial z}-r_{1}\left[\left(2 q_{2} \frac{\partial^{2}}{\partial z^{2}}+\lambda_{2}\right)+\frac{2}{r_{2}^{2}}\right] U_{z, 1}=0 .
\end{gathered}
$$

Here $r_{1}, r_{2}-$ are inner and outer radii of the shell, $r_{2}>r_{1} ; \mu$ is shear coefficient of the shell material; $U_{r, 0}$ and $U_{r, 1}$ are the main part of the radial displacement of shell points; $U_{z, 0}$ and $U_{z, 1}$ are the main part of the longitudinal displacement of shell points; $a_{0}$ is sound velocity in a fluid at rest; $\rho_{0}^{\prime}-$ is plane fluid at rest; $\mu^{\prime}-$ is coefficient viscosity fluid; $v^{\prime}-$ is kinematic coefficient viscosity fluid;

$$
\begin{aligned}
& q_{1}=-\frac{1}{1-2 v}, q_{2}=-\frac{1}{2(1-v)}, \lambda_{1}^{m}=\left(\frac{1}{a^{2}} \frac{\partial^{2}}{\partial t^{2}}-\frac{\partial^{2}}{\partial z^{2}}\right)^{m}, \lambda_{2}^{m}=\left(\frac{1}{b^{2}} \frac{\partial^{2}}{\partial t^{2}}-\frac{\partial^{2}}{\partial z^{2}}\right)^{m}, \\
& m=0,1,2, \ldots
\end{aligned}
$$

where: $\mathcal{V}-$ is Poisson's coefficient; $a, b$ art the propagation velocity of longitudinal and transverse waves in the shell material, respectively.

The obtained equations of oscillation (1), in particular cases, go over to the classical equation of longitudinal vibrations of a circular bar, to the Rayleigh equation, or to the refinement Timoshenko-type equation. They can be used to solve applied problems of the interaction of a cylindrical shell with a viscous compressible fluid. With the vibration equations, formulas are derived for all nonzero components of the strain tensor and displacement vector. For instance,

$$
\begin{aligned}
& \sigma_{z z}(r, z, t)=\mu\left\{\begin{array}{l}
\left(1-q_{1}\right) \frac{\partial U_{z, 0}}{\partial z}-\left(1+q_{1}\right) U_{r, 0}- \\
-r_{1}\left(\ln \frac{r}{r_{1}}+\frac{1}{2}\right)\left[\left(1-2 q_{2}\right) \frac{\partial U_{z, 1}}{\partial z}+\left(1+2 q_{2}\right) \lambda_{2} U_{r, 1}\right]
\end{array}\right\}, \\
& \sigma_{r r}(r, z, t)=-\mu\left\{\left[q_{1} U_{r, 0}+\left(1+q_{1}\right) \frac{\partial U_{z, 0}}{\partial z}\right]+r_{1}\left\{\left[\left(q_{2} \ln \frac{r}{r_{1}}-\frac{1}{2}\right) \lambda_{2}+\frac{2}{r^{2}}\right] U_{r, 1}+\right.\right. \\
& \left.+\left[\left(1+q_{2}\right) \ln \frac{r}{r_{1}}+\frac{1}{2}\right] \frac{\partial U_{z, 1}}{\partial z}\right\} \\
& U_{z}(r, z, t)=U_{z, 0}-\left(\ln \frac{r}{r_{1}}+\frac{1}{2}\right)\left(r_{1} U_{z, 1}\right) \text {, }
\end{aligned}
$$




$$
U_{r}(r, z, t)=\frac{r}{2}\left\{U_{r, 0}-r_{1}\left[\left(1+q_{2}\right) \lambda_{2} \ln \frac{r}{r_{1}}+\frac{2}{r^{2}}\right] U_{r, 1}-r_{1} q_{2} \ln \left(\frac{r}{r_{1}}\right) \frac{\partial U_{z, 1}}{\partial z}\right\}
$$

For the correct formulation of the boundary conditions of applied problems, when truncating the number of terms in the series of equations (1), one should adhere to the same accuracy as in the oscillation equations.

\subsection{Statement of the problem}

We study the process of longitudinal deformation of a semi-infinite elastic circular cylindrical shell filled with a viscous compressible fluid excited by a suddenly included longitudinal displacement at the end $z=0$. To solve the problem, for the main resolving equations, we take the specified equations of the longitudinal radial vibrations of a circular cylindrical shell with a viscous compressible fluid. To obtain such an equation, we use equations (1). Considering purely longitudinal vibrations, we can assume that the radial displacement (and its main parts) is equal to zero. This condition can be achieved, for example, by dressing a rigid ring that is not deformed in the radial direction on end face subjected to an external load. Then, the equations of longitudinal vibrations of the cylindrical shell obtained from (1), taking into account the above remarks and in dimensionless variables, will have the form

$$
\begin{gathered}
{\left[\frac{2-2 v}{1-2 v} \cdot\left(\frac{b^{2}}{a^{2}} \frac{\partial^{2}}{\partial t^{2}}-\frac{\partial^{2}}{\partial z^{2}}\right)-\frac{b^{2} \rho_{0}^{\prime}}{r_{1} \mu}\left(\frac{a_{0}^{2}}{b^{2}} \frac{\partial^{2}}{\partial z^{2}}+\frac{4 v^{\prime}}{3 b r_{1}} \frac{\partial^{3}}{\partial t \partial z^{2}}-\frac{\partial^{2}}{\partial t^{2}}\right)\right] U_{z, 0}-} \\
-2\left[1+\frac{b^{2} \rho_{0}^{\prime}}{4 r_{1} \mu}\left(\frac{a_{0}^{2}}{b^{2}} \frac{\partial^{2}}{\partial z^{2}}+\frac{4 v^{\prime}}{3 b r_{1}} \frac{\partial^{3}}{\partial t \partial z^{2}}-\frac{\partial^{2}}{\partial t^{2}}\right)\right] U_{z, 1}+\frac{2 b \rho_{0}^{\prime}}{3 r_{1}^{2} \mu} \cdot \frac{\partial^{2} U_{z, 1}}{\partial t^{2}}=0 ; \\
\left(\frac{b^{2}}{a^{2}} \frac{\partial^{2}}{\partial t^{2}}-\frac{\partial^{2}}{\partial z}\right) U_{z, 0}-\frac{r_{1}^{2}}{r_{2}^{2}} \frac{1-2 v}{(1-v)} U_{z, 1}=0 .
\end{gathered}
$$

Where the fluid reaction is equal

$$
R=\frac{b^{2} \cdot \rho_{0}^{\prime}}{r_{1} \mu}\left(\frac{a_{0}^{2}}{b^{2}} \frac{\partial^{2}}{\partial z^{2}}+\frac{4 v^{\prime}}{3 b r_{1}} \frac{\partial^{3}}{\partial t \partial z^{2}}-\frac{\partial^{2}}{\partial t^{2}}\right)
$$

In the expression for the fluid reaction (4), the second term is responsible for the reaction of the fluid due to viscosity. In this case, the kinematic viscosity coefficient for most fluids is of the order $10^{-4}$ and below. In addition, the Lame coefficient present in the denominator of the coefficient of this term is of the order $10^{10}$ and higher. Therefore, we can assume that the contribution of the second term in (3.2) is very small, and it can be neglected. Then the boundary conditions of the problem recorded for the main part of the longitudinal displacement will have the form 


$$
U_{z, 0}-\frac{1}{2} U_{z, 1}=g(t)_{\text {at }} z=0, \quad U_{z, 0}=U_{z, 1}=0 \text { at } z \rightarrow \infty,
$$

where $g(t)$ is impulse of a given displacement. The initial conditions are assumed to be zero.

\subsection{Solving of the problem}

We assume that the displacements of the cylindrical shell have sufficiently small quantities. Then the main parts of the displacement $U_{z}$ and their derivatives of the third and higher orders will be small quantities of a higher order. Therefore, in the first equation (1), we neglect the derivatives of third-order functions, and solving the system with respect to $U_{z, 1}$ we will have

$$
\frac{\partial^{2} U_{z, 0}}{\partial t^{2}}=2 \frac{1-v}{1-2 v} \frac{\partial^{2} U_{z, 0}}{\partial t^{2}}+\frac{1}{r_{2}^{2}-1}\left[\theta_{1} \frac{\partial^{2}}{\partial z^{2}}-\theta_{2} \frac{\partial^{2}}{\partial t^{2}}\right] U_{z, 0}=0
$$

Where: $\theta_{1}=\frac{a_{0}^{2} \rho_{0}^{\prime}}{r_{1} \mu} ; \quad \theta_{2}=\frac{b^{2} \rho_{0}^{\prime}}{r_{1} \mu}$.

Where in

$$
U_{z, 1}=\frac{r^{2}}{2}\left(1-q_{1}\right)\left(\frac{b^{2}}{a^{2}} \frac{\partial^{2}}{\partial t^{2}}-\frac{\partial^{2}}{\partial z}\right) U_{z, 0} .
$$

To solve equation (6), we apply the Laplace transform. Then the general solution (6), due to (7), has the form

$$
\begin{gathered}
\bar{U}_{z, 0}=A e^{-\alpha z}, \alpha=\sqrt{\frac{a_{2}}{a_{1}+b_{0}^{2}}} p ; \\
b_{0}^{2}=\frac{2-2 v}{1-2 v} ; \quad a_{1}=\frac{\theta_{1}}{r^{2}-1} ; \quad a_{2}=\frac{\theta_{2}}{r^{2}-1}-1 .
\end{gathered}
$$

In case of the absence of fluid takes place $\alpha=p / b_{0}$.

Based on (7), we find

$$
\bar{U}_{z, 1}=A \frac{r_{2}^{2}}{2} \frac{a_{1}-b_{0}^{2}\left(1+a_{2}\right)}{a_{1}+b_{0}^{2}} p^{2} e^{-\sqrt{\frac{a_{2}}{a_{1}-b_{1}^{2}}} p z}
$$

Then according to formulas (2) for the transformed $\bar{U}_{z}$ we get the expression 


$$
\bar{U}_{z}=A\left[1-\frac{r^{2}}{4} \frac{a_{1} b_{0}^{2}\left(1+a_{2}\right)}{a_{1}+b_{0}^{2}} p^{2}\right] \cdot e^{-\sqrt{\frac{a_{2}}{a_{1}-b_{0}^{2}}} p z}
$$

Substituting the obtained expression into the Laplace-transformed boundary conditions (5) for constant integration $-A$, we have

$$
A=\frac{a_{3}^{2} G(p)}{p^{2}+a_{3}^{2}},
$$

where: $a_{3}^{2}=\frac{4 \beta^{2}}{r^{2}\left[-a_{1}+b_{0}^{2}\left(1+a_{2}\right)\right] a_{2}} ; \beta^{2}=\frac{a_{2}}{a_{1}+b_{0}^{2}}$.

Then, putting the expression $A$ into (8) and (9), followed by access to the parameter $p$ , get the solution to the problem

$$
\begin{gathered}
U_{z, 0}=a_{3} \int_{\beta z}^{t} g(\xi-\beta z) \sin \left[a_{3}(t-\xi)\right] d \xi \\
U_{z, 1}=2 a_{3} \int_{\beta z}^{t} g(\xi-\beta z) \sin \left[a_{3}(t-\xi)\right] d \xi-2 g(t-\beta z) .
\end{gathered}
$$
form

Now it is easy to calculate strains based on formulas (2), which in our case have the

$$
\begin{gathered}
\sigma_{z z}(r, z, t)=\mu\left\{\left(1-q_{1}\right) \frac{d U_{z, 0}}{d z}+\frac{1-2 q_{2}}{2} \frac{d\left(r_{1} U_{z, 1}\right)}{d z}\right\} \\
\sigma_{r r}(r, z, t)=-\mu\left(1+q_{1}\right) \frac{d U_{z, 0}}{d z}+\frac{\mu r_{1}}{2} \frac{d U_{z, 1}}{d z} .
\end{gathered}
$$

It is easy to obtain formulas for stresses passing here to dimensionless variables and then substituting expressions (3.10) into the obtained formulas. For instance:

$$
\begin{aligned}
& \frac{\sigma_{z z}}{\mu}=q_{1} a_{3} \beta\left[\int_{\beta z}^{t} g^{\prime}(\xi-\beta z) \sin \left[a_{3}(t-\xi)\right] d \xi+\frac{g(0)}{a_{3}} \sin \left[a_{3}(t-\beta z)\right]\right]- \\
& -2\left(1-2 q_{2}\right) \beta g^{\prime}(t-\beta z), \\
\frac{\sigma_{r r}}{\mu}= & \beta g^{1}(t-\beta z)+q_{1} \beta a_{3}\left[\int_{\beta z}^{t} g^{\prime}(\xi-\beta z) \sin \left[a_{3}(t-\xi)\right] d \xi+\frac{g(0)}{a_{3}} \sin \left[a_{3}(t-\beta z)\right] .\right.
\end{aligned}
$$

Formulas (12), (14), and (15) allow us to calculate the longitudinal displacement and strains at an arbitrary point of the shell, taking into account the fluid contained in it, i.e., to determine the stress-strain state of an arbitrary section of the hydroelastic system under consideration. 


\section{Results and Discussions}

To numerically study the strains and determine the influence of fluid and changes in its density on them, they were calculated using formulas (3.12) and (3.13). It is believed that the shell material is steel with characteristics $v=0.3$ and $E=2 \cdot 10^{11} \Pi a$. In this case, the sound propagation velocity in the fluid is taken as equal to $a_{0}=1500 \mathrm{M} / \mathrm{c}$, for dimensionless fluid density in the above formulas, different values are accepted from 200 to 500. The calculations are made for the external load specified by the formula $g(t)=g_{0} \sin \left(\pi t / t_{1}\right)$. The calculation results are shown in Fig. 2-5. Figures 2 and 3 show the graphs of changes in radial strain depending on the longitudinal coordinate for a fixed value of time $\mathrm{t}=5,\left(\mathrm{t}_{1}=2\right)$. From the above dependencies, it follows that the strain $\sigma_{r r}$ и $\sigma_{z z}$ strongly dependent on fluid density. For example, in the cross-section $z=0$ strain value $\sigma_{r r}$ (Fig.3) at $\rho_{0}^{\prime}=200$ (curve 1) 5.5 times exceeds it, value at $\rho_{0}^{\prime}=1500$ (curve 4 ) and by $53 \%$ exceeds it for $\rho_{0}^{\prime}=1000$ (curve 3 ). In addition, the presence of a fluid, even without taking into account the kinematic viscosity, leads to rapid damping of the strain $\sigma_{r r}$ along the longitudinal coordinate. For example, strain $\sigma_{r r}$ in cross-section $z=11$ drops 3.75 times compared with the cross-section $z=0$ (curve 3). A similar picture takes place in the case of normal longitudinal stresses (Fig. 3). But, here, the effect of fluid density is manifested to an even greater extent.

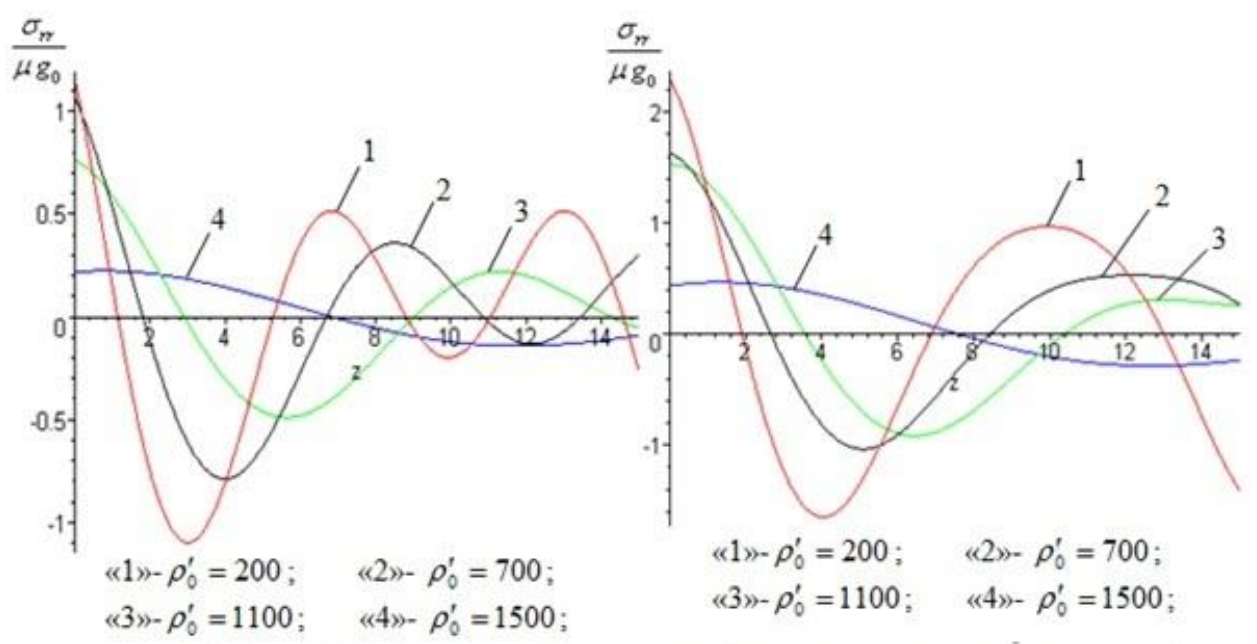

Fig.2. Change of radial stress depending on longitudinal coordinates with fixed value of time $t=5, t_{1}=2$.

Fig.3. Change of longitudinal stress depending on longitudinal coordinates with fixed value of time $t=5, t_{1}=2$. 


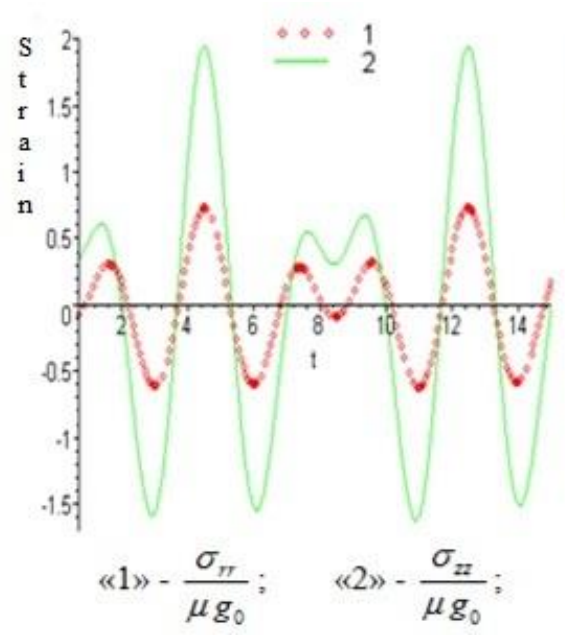

Fig.4. Comparison of changes of stresses $\sigma_{r r}$ and $\sigma_{z z}$ by time $t$ on cross-section $z=1.5$ at $t=2$.

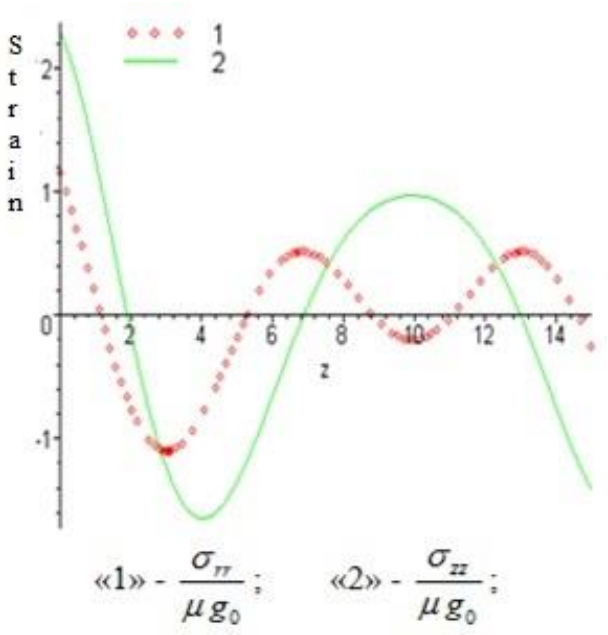

Fig.5. Comparison of changes of stresses $\sigma_{r r}$ and $\sigma_{z z}$ by coordinate $z$ at $t_{1}=2 ; t=5$.

For example, in section $z=0$ strain value $\sigma_{r r}$ (Fig. 3) at $\rho_{0}^{\prime}=200$ (curve 1) almost 10 times (against 5.5 times in the case of strain $\sigma_{r r}$ ) exceeds its value at $\rho_{0}^{\prime}=1500$ (curve 4) and by $72 \%$ (against 53\%) exceeds it at $\rho_{0}^{\prime}=1000$ (curve 3). Strain damping $\sigma_{r r}$ along the longitudinal coordinate even faster. For example, strain $\sigma_{\text {яя }}$ in the cross section $z=13$ drops by almost 5 times (versus 3.75) compared with the cross-section $z=0$ (curve 3 ).

Conducted to verify the reliability of the obtained results, a comparative analysis is showed

that the refined equations of axisymmetric vibrations of the hydroelastic system under consideration, in the particular case of the absence of an interacting viscous fluid, coincide in structure but differ in coefficients with the corresponding results of [ 8,21].

The proposed approximate equations of longitudinal vibrations of elastic circular cylindrical shells interacting with a viscous fluid are proposed, suitable for solving applied engineering practice problems.

\section{Conclusions}

- strains $\sigma_{r r}$ and $\sigma_{z z}$ at the points of the hydroelastic system under consideration, they strongly depend on the density of the fluid: with an increase in the density of the fluid, the strain values rapidly decrease, reaching a 5.5-fold decrease in the case of strain $\sigma_{r r}$ and 10 multiple reduction in the case $\sigma_{z z}$ in cross-section $z=0$;

- strain intensively attenuates along with the distance along the longitudinal coordinate. Values of the normal longitudinal stress in the cross-section $z=13$ decrease by 5 times compared with the cross-section $z=0$; Similarly, in the case of normal radial stresses, it decreases by 3.5 times compared with the same zero cross-section. 


\section{References}

1. Herrmann G., Mirsky I. Journal Applied Mechanics 23 (4), pp. 563-568. (1956)

2. Grigolyuk E.I., Selezov I.T. Itogi nauki i techniki. Series Mechanika deformiruyemogo tverdogo tela, Results of science and technology Series Deformable Solid Mechanics, 5 Moskov: VINITI, (1973)

3. Nigul U.K. Teoriya plastin $i$ obolochek, theory of plates and shells, pp. $738-742$. Yerevan (1964)

4. Netrebko A.V., Pshenichnov S.G. Izvestiya Tulskogo gosudarstvennogo universiteta, Estestvennyie nauki, Bulletin of the Tula State University. Natural Sciences, 4 pp. 6379.

5. Netrebko A.V., Pshenichnov S.G. Problemy prochnosti i plastichnosti, Problems (2014) of strength and ductility 77 (1), pp. 67-74, (2015)

6. Khudoynazarov Kh, Khalmuradov R.I., Yalgashev B.F, Vestnik Tomskogo gosudarstvennogo universiteta. Matematika i Mekhanika, Bulletin of the Tomsk State University.Mathematika and Mechanica, 69 pp. 139-154. (2021), doi: 10.17223/19988621/69/11.

7. Petrashen G.I., Studies on elasticity and plasticity, Jeningrad: Publishing house of LGU, 5, pp. 3-33, (1966)

8. Filippov I.G., Kudainazarov K. Soviet Applied Mechanics 26 (2), pp. 161-168, (1990), doi:10.1007/bf00887110.

9. Filippov I.G., Kudainazarov K. International Applied Mechanics 34(12) pp. 1204 1210. (1998), doi:10.1007/bf02700874.

10. Khudoynazarov Kh., and Khudoyberdiyev Z.B., IOP Conf. Ser.: Earth Environ. Sci. 614 p. 012061 (2020), doi:10.1088/1755-1315/614/1/012061.

11. Khudoynazarov Kh and Yaxshiboyev Sh.R. IOP Conf. Ser.: Earth Environ. Sci. 614 012062. (2020), DOI: 10.1088/1755-1315/614/1/012062.

12. Khudoynazarov K. IOP Conf. Series: Mater. Sci. Eng. p. 1030 p. 012098 (2021), doi: 10.1088/1757-899X/1030/1/012098.

13. Khalmuradov R.I., Yalgashev B.F., IOP Conf. Ser.: Earth Environ. Sci. 614, p. 012087. (2020) doi: 10.1088/1755-1315/614/1/012087.

14. R.I.Khalmuradov and E.A.Ismoilov 2020 IOP Conf IOP Conf. Ser.: Earth Environ. Sci. 614 012071. DOI: 10.1088/1755-1315/614/1/012071.

15. Khalmuradov R.I. Uzbek Journal Problembl of Mechanics 2-3 pp. 53-57, (2017)

16. Khalmuradov R.I. 2017 Scientific Journal of Samarkand university 3, pp. 86-90, (2017)

17. Grigolyuk E.I. Gorsgkov A.G. Interaction of elastic structures with fluid, Hit and dive, Leningrad, (1976)

18. Guz A.H. 1980 Soviet Applied Mechanics 16 (10), pp. 10-20.

19. Abassi W., Baroudi A.E. Razafimahery F. International Journal of Applied Mechanics February (2016) doi: 10.1142/S1758825116500320.

20. Xuebin Li. Journal of Sound and Vibration 311(3-5) pp. 667-682. (2008) DOI:org/10.1016/j.jsv.2007.09.023.

21. Khudoynazarov Kh. Proceedings of the 8th Int. Conference on Shell Structures: Theory and Applications. Gdansk-Jurata, pp. 343-347. Poland, Balkema, London, (2005) 